\title{
CT Angiography in the Preoperative Evaluation as a Predictor of Surgical Time for DIEP
}

\author{
Moraes Pithon Napoli JV, ${ }^{1 *}$ Maria Eduarda Penachio Sarmento, ${ }^{2}$ Gabriela Ducioni Matos $^{3}$ \\ ${ }^{1}$ University of Western Australia / Universidade nove de julho, Australia/Brazil \\ ${ }^{2}$ Universidade Municipal de São Caetano do Sul, Brazil \\ ${ }^{3}$ Universidade Nove de Julho, Brazil
}

\begin{abstract}
Computed tomography angiography (CT-angiography) provides a clear view of the perforating vessels and their course, being essential in the preoperative evaluation of mastectomies. In the case of DIEP (deep lower epigastric artery), the prior identification of these vessels is of great importance for breast reconstruction, because in addition to being a predictor of surgical time, it helps in the correct approach of the arteries.
\end{abstract}

Objective: To analyze CT angiography as a predictor of the surgical time for DIEP, when performed in the preoperative evaluation.

Methods: A database from the Royal Perth Hospital in Australia was analyzed, with a total of 104 mastectomies performed during the period from January to December 2017. Among them, 19 were for breast reconstruction by DIEP. The patients had an average of 47 years, ranging from 31 to 69 years.

Results: We obtained that when the average number of arteries in the CT angiography is less than four, there will be an average decrease of 34 minutes in the total time of surgery. However, if the number is greater than or equal to five arteries, there will be an average increase of 34 minutes, totaling an average of 452 and 551 total minutes of surgery, respectively.

Conclusion: Angio-CT is an important predictor to predict the average time of surgery, one of the main factors related to complications when prolonged.

Keywords: CT angiography, DIEP, Mastectomy, Duration, Microsurgery.

\section{Introduction}

Breast cancer is the most common cancer in women worldwide. In 2018, there were about 2.1 million new cases, accounting for $24.2 \%$ of the total estimated cases. In Brazil, breast cancer is also the most common neoplasm, with the exception of non-melanoma skin tumors. The National Cancer Institute (INCA-20) estimated 66,280 new cases in the country for each year of the 2020-2022 triennium, with an estimated risk of 61.61 new cases per 100,000 women. ${ }^{1}$

Despite great advances, mastectomy is still the most frequent treatment and is used even for prophylaxis of malignancies. ${ }^{2}$ However, this procedure can evolve with biopsychosocial complications, which negatively influence the patient's quality of life and self-esteem. $^{3}$

As a result, there is an increasing demand for breast repair surgery, in search of better acceptance of self-image. In 2018, the American Society of Plastic Surgery registered 101,657 breast reconstructions performed in the United States, about $29 \%$ more procedures than in 2000. ${ }^{4}$ In Brazil, according to the Goiana Research Network, approximately 92,500 mastectomies were performed between the years 2005 and 2018, of these 18,000 (20\%) were reconstructed by the Unified Health System. ${ }^{5}$

There are several breast reconstruction techniques, which are used both in mastectomized patients and also in malformations.
Quick Response Code:

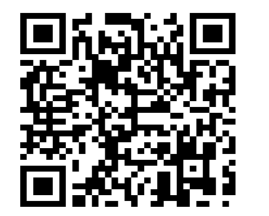

*Corresponding author: João Vitor Moraes Pithon Napoli, Universidade nove de julho, rua pamplona 1119, Brazil

Received: 06 May, 2021

Published: 27 May, 2021

Citation: Moraes PNJV, Maria EPS, Gabriela DM. CT Angiography in the Preoperative Evaluation as a Predictor of Surgical Time for DIEP. Mod Res Plast Recon Sur. 2021;1(2):1-5. DOI: 10.53902/MRPRS.2021.01.000506 
The techniques range from the use of local flaps (eg, plug flap), mammoplasty techniques, autologous flaps, use of alloplastic materials (eg, prostheses, expanders) to mixed techniques. (2) These methods keep updating with time and are used to simulate the volume and shape of the breast, and should be chosen according to each case. ${ }^{6}$

According to the American Society of Plastic Surgeon Statistics, in 2016, the most used procedure for breast reconstruction remains the use of an expander. However, since it is alloplastic material, there is a higher incidence of rejection and some more aesthetically artificial results. ${ }^{7}$

Among the autologous techniques, in the past, the thoracodorsal muscle-skin flap, abdominal flap, and tubular skin flap were used a lot. However, its aesthetic result was generally unsatisfactory, due to the lack of volume and often limited skin extension.

With the evolution of microsurgery, a technique emerged in the 1970s that is still one of the main options, the transverse rectus abdominis muscle (TRAM) flap. This technique has the advantage of not requiring the use of prostheses for volumizing and also allowing the improvement of abdominal appearance. However, loss of sensation due to the innervation of the transplanted tissue and abdominal wall injuries, such as hernias, were common in this technique. $^{8}$

It was then that in 1989, Koshima and Soeda described a technique that also used an abdominal flap, but preserving the abdominal musculature, called the inferior epigastric artery perforating flap (DIEP). This method is currently considered the gold standard for breast reconstruction (18). ${ }^{9}$ It consists of the use of an abdominal pedicled flap of only skin and adipose panicle, without fascia or muscle, which is irrigated by one or two arterial pedicles, originating in the lower epigastric arteries and main perforating branches. 6

When compared, DIEP offers less postoperative pain to the patient, less morbidity, less recovery time, less postoperative hospital stay, lower cost 10 , higher satisfaction rates with the aesthetic result and still preserves the muscle wall of the donor area, with a lower incidence of hernias and bulging of the abdominal wall compared to TRAM. ${ }^{10-12}$

However, DIEP has risks, mainly due to the injury of intraoperative vessels. Beugels, et al. in 2018, in his study comparing immediate and late complications of DIEP, he concluded that the most common complications were the least, with fatty necrosis being the most common, followed by hematoma, wound problems, infection and seroma. The least common were the largest, the most common being venous congestion, followed by partial loss of the flap and, rarely, total loss of the flap. ${ }^{13}$

The DIEP technique initially consists of preparing the flap, which begins with an elliptical incision in the lower abdomen, between the umbilicus, the pubic symphysis, and the iliac spine. Then, viable perforating arteries to be dissected must be analyzed and selected, preference is given to perforators that penetrate the muscle at the level of the tendon intersections.
After this atraumatic microsurgical resection of the arterial pedicle of the fibers of the rectus abdominis muscle. The donor area is corrected using abdominoplasty techniques. The receiving area, on the other hand, is prepared to receive the flap, and the internal thoracic artery is sectioned to perform an end-to-end anastomosis with the arterial pedicle of the flap. ${ }^{14}$ For venous drainage of the flap, breast vessels are sought at the third costochondral junction. ${ }^{15}$

As it is a highly complex technique, it ends up needing a longer operating time, material suitable for microsurgery, and extensive anatomical knowledge of the area. Few professionals are qualified to perform it with skill, and their learning curve is long, taking years for an adequate improvement. Laportaet al. in 2017, he related in his article about time-dependent factors in breast reconstruction with the DIEP flap the question of the learning curve and a number of perforating arteries interfering with the surgical time $(\mathrm{p}<0.005)$. Removing in this same article the size of the flap as interference. ${ }^{16}$

Furthermore, DIEP is not seen as a good option for very thin women who do not have enough extra subcutaneous tissue in the abdomen, women who have undergone other abdominal surgeries, including colostomy or abdominoplasty and women whose abdominal blood vessels are small or not are in the best location for the flap. ${ }^{17}$

To assess the viability and anatomical variations of the donor region, some places already use studies of local vascularization in the preoperative assessment, identifying the perforating arteries suitable for the procedure. This allows for better planning of the surgery, and consequently a reduction in intraoperative time and complications. This study of the preoperative patient is relevant since the knowledge of the location of the lower epigastric artery is consistent in the literature, but of the perforating arteries, it varies drastically from individual to individual, making its location and selection difficult. ${ }^{18}$

The mapping of the region can be done by ultrasonography (USG) doppler, color USG Doppler, computed tomography angiography (CT angiography), and magnetic resonance angiography.19 USG doppler is the most common method used, as it is a more accessible and cheaper tool. However, it is extremely sensitive, and not only shows clinically relevant perforating arteries $(>1 \mathrm{~mm})$, but also extremely small perforating arteries. Its accuracy is not ideal for the study of preoperative vascular anatomy. ${ }^{20}$

CT angiography, on the other hand, is considered the best cost-benefit, as it provides a clear visualization of the perforating vessels previously, being fundamental for the subsequent breast reconstruction and enabling the correct approach of the arteries. ${ }^{21}$ Without these methods, the location of these vessels is done intraoperatively through the visualization of the arterial diameter and palpation of the pulse, which takes much longer and does not always opt for the best available vessel (20).

Malhotra, et al. in 2012, in his studies on DIEP flap guided by CT angiography, he demonstrated that with better preoperative planning, the operative time was reduced, also reducing the number of patients who needed blood transfusions. ${ }^{22}$ 
It was also demonstrated in a study on the financial implications of CT angiography in DIEP conducted in 2009 by Rozen, et al., that preoperative CT angiography reduces on average 90 minutes of each surgery, with a margin of error of 34 minutes more in the case of more than 5 perforators, and less in the case of fewer than 5 perforators. ${ }^{23}$ In the same study, they compared the costs for performing a CT angiography with the costs of 90 minutes more surgery, reaching a number up to 6 times higher.

In this context, this study analyzed one of the Royal Perth Hospital databases in 2017, in order to understand CT angiography as a predictor of the surgical time for DIEP, when performed in the preoperative evaluation.

\section{Materials and Methods}

The data presented by the patients of the Royal Perth Hospital - Department Division of Plastic Surgery, in Australia, between January and December 2017, were used as numerical materials in this article. The patients in the present study were aged between 31 and 69 years old, with an estimated average of 47 years old. Inclu- sion criteria for this study were considered: mastectomized female patients, between 31 and 69 years old, who underwent breast reconstruction at the Royal Perth Hospital from January to December 2017, after a preoperative AngioTC. Among them, a total of 104 mastectomies were evaluated, with $22 \%$ unilateral mastectomy with surgical reconstruction, $14 \%$ bilateral mastectomy also with surgical reconstruction, and 64\% mastectomy without presenting any reconstruction.

According to these data, of the total number of mastectomized patients, in 19 patients able to breast reconstruction, the DIEP technical flap was used, in which they underwent preoperative planning, obtained by the radiological study of the perforating arteries provided by the rich analysis. in detail of the contrasted CT-Angio. In addition, they were subjected to an intraoperative evaluation in order to assess the surgical time and any complications or specific surgical variable in each one, and finally, the postoperative study, in order to monitor possible complications arising from the surgery. And assess the general condition of the operated patient as a whole(Figure 1).

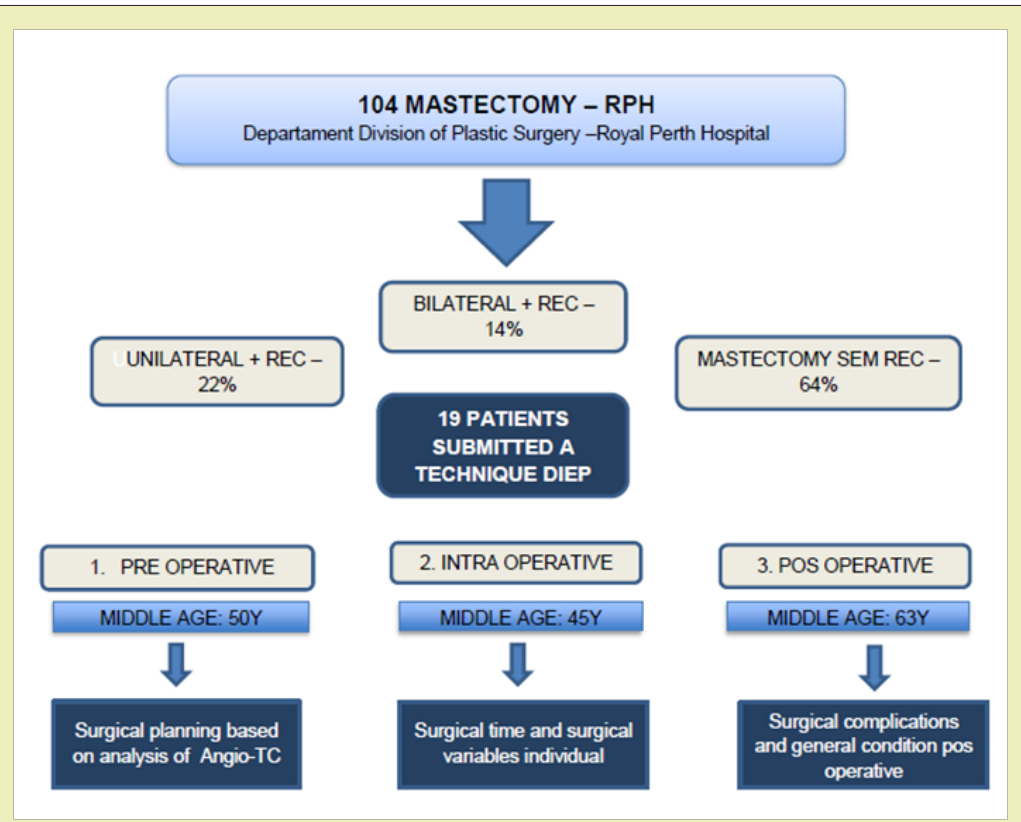

Figure 1: 104 Mastectomy RPH.

\section{Results}

\section{Population}

According to the findings within the inclusion criteria, it was observed that 104 mastectomies were performed from January to December 2017. Among them, 19 were for breast reconstruction by DIEP. The patients had an average of 47 years, ranging from 31 to 69 years.

\section{Preoperative angio-CT assessment}

The results of the preoperative evaluation with Angio-CT indicated that the average number of perforating arteries presented by the patients was 5.3 , in which $41 \%$ had a good caliber, $24.4 \%$ moderate, and $34.6 \%$ small caliber(Figure 2).

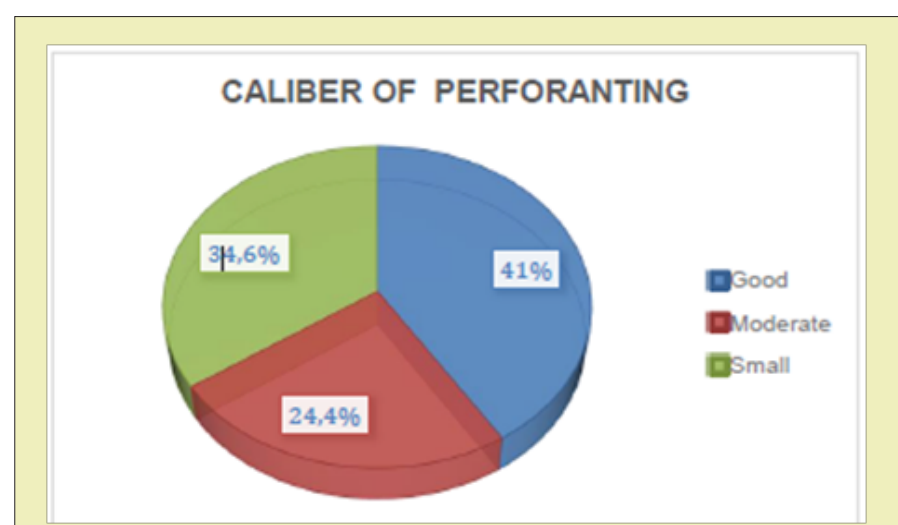

Figure 2: Caliber of Perforanting. 


\section{Surgical time}

Regarding the surgical time, it was observed that when the average number of arteries on CT angiography was less than four, there was an average reduction of 34 minutes in the total time of surgery. However, when the number was greater than or equal to five arteries, there was an average increase of 34 minutes, totaling an average of $482(\mathrm{p}<0.01)$ and 551 total minutes of surgery, respectively. The mean surgical time was 517 minutes $(\mathrm{p}<0.05)$ (Figure 3).

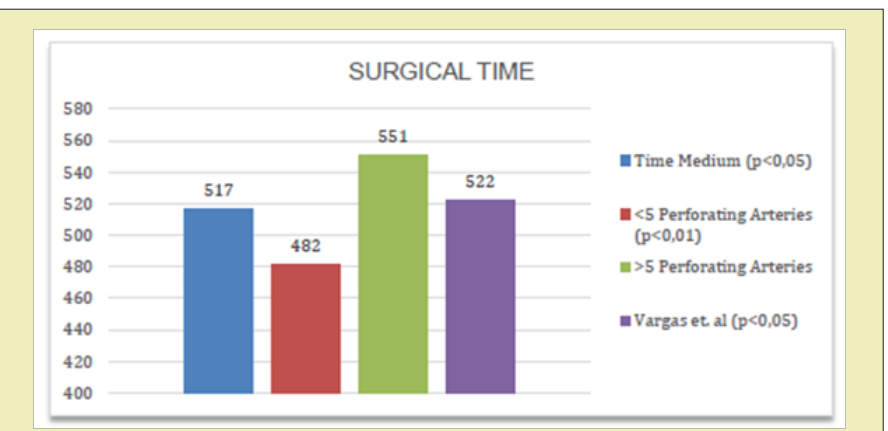

Figure 3: Surgical time.

\section{Flap size}

The average volume of the removed flaps was $1206 \mathrm{~mm}^{3}$. However, the flap size did not interfere with the surgical time.

\section{Complications}

In $31 \%$ of cases, some complication of the minor criteria was observed, such as postoperative infection, dehiscence, use of the cephalic vein, intraoperative vessel injury, and postoperative hematoma. No case was observed with major criterion complications.

\section{Conclusion}

It is concluded, therefore, based on the data provided in this study, that the use of preoperative CT angiography to evaluate perforating arteries, in order to use them as a basis for the technical application DIEP for breast reconstruction of patients undergoing mastectomy, it is essential, in order to reduce surgical time. With less than four perforating arteries seen on radiological examination, there is a 34-minute reduction in surgical time, whereas, in the presence of five or more arteries, the total time of surgery is increased by 34 minutes.

\section{Acknowledgments}

None.

\section{Funding}

None.

\section{Conflicts of Interest}

Author declares that there is no conflict of interest.

\section{References}

1. INCA (2021) Conceito e Magnitude do câncer de mama.
2. Leonardo David Pires B, Di Lamartine Galdino Amara J, Carlos Daher J, et al. Breast reconstruction with a latissimus dorsi myocutaneous flap and implants: evaluation of strategies adopted to improve the results and evaluation pelo Breast - Q. Revista Brasileira de Cirurgia Plástica. 2018;33(4):453-462.

3. Ognev Meireles C, Pedro Pontes Camara Filho J, de Souza Honorato de Barros AG, et al. Breast reconstruction procedures: a 10-year retrospective study. Rev Bras Cir Plast. 2013;28(1):59-64.

4. ASPS. National Clearinghouse of Plastic Surgery Procedural Statistics Report. 2018.

5. Only $20 \%$ of women had their breasts reconstructed in Brazil between 2008 and 2015.

6. Webster RS, Goldoni BD, Netto R, et al. Breast reconstruction with perforator flaps: use of the DIEAP flap at the Plastic Surgery and Microsurgery Service of Universidade Federal de Ciências da Saúde de Porto Alegre and Santa Casa de Misericórdia de Porto Alegre. Rev Bras Cir Plást. 2014;29(3):384-389.

7. Andejani DF,AlThubaitiGA. Intersection-splitting Deep InferiorEpigastric Perforator Flap. Plast Reconstr Surg Glob Open. 2019;7(10):e2490.

8. Doncatto L, Caleffi M. Breast Reconstruction With Sensitive Tram Flap Reinnervation. Rev Bras Cir Plást.1997;12(2):35-46.

9. Risal Djohan, James E Zins, David K. et al. Rolston Options and considerations in the timing of breast reconstruction after mastectomy. Cleveland Clinic Journal of Medicine. 2008;75(3 Suppl 1):S30-S33.

10. Smit JM, Dimopoulou A, Liss AG, et al. Preoperative CT angiography reduces surgery time in perforator flap reconstruction. J Plast Reconstr Aesthet Surg. 2009;62(9):1112-1117.

11. Barbara d'Ávila Goldoni1, Paulo Solano Júnior, Geraldo Cesar Castro Althof, et al. Breast reconstruction using deep inferior epigastric perforator (DIEP) flap - review of the literature and case report. Arquivos Catarinenses de Medicina. 2014;43(Suppl 1):104-107.

12. Ono MCC, Groth AK, Silva ABD, et al. Recipient vessel options in microsurgical breast reconstruction. Rev Bras Cir Plást. 2013;28(2):227232.

13. Beugels J, Bod L, van Kuijk SMJ, et al. Complications following immediate compared to delayed deep inferior epigastric artery perforator flap breast reconstructions. Breast Cancer Res Treat. 2018;169(2):349-357.

14. Ulatowski Ł, Kaniewska A. The Use of The Diep Flap In The Modern Reconstructive Surgery. Pol Przegl Chir. 2015;87(9):472-81.

15. Blondeel PN. One hundred free DIEP flap breast reconstructions: a personal experience. British Journal of plastic surgery. 1998;52(2):104111.

16. Laporta R, Longo B, Sorotos M, et al. Time-dependent factors in DIEP flap breast reconstruction. Microsurgery. 2017;37(7):793-799.

17. Kaplan JL, Allen RJ. Cost-based comparison between perforator flaps and TRAM flaps for breast reconstruction. Plast Reconstr Surg. 2000;105(3):943-948.

18. Yeonhoon Lee, Sung Chan Kim, Jin Sup Eom, et al. Classification of Deep Inferior Epigastric Perforator Courses Based on Computed Tomography Angiography: Incidences and Clinical Implications. Archives of Hand and Microsurgery. 2018;23(4):281-289.

19. Ulatowski $Ł$, Kaniewska A. The Use Of The Diep Flap In The Modern Reconstructive Surgery. Pol Przegl Chir. 2015;87(9):472-81.

20. Mohan AT, Saint-Cyr M. Advances in imaging technologies for planning breast reconstruction. Gland Surg. 2016;5(2):242-254.

21. Cunha MS, Munhoz AM, Sturtz G, et al. Evaluation of the inferior epigastric artery perforator flap perfusion in breast reconstruction. Rev Bras Cir Plást. 2006;21(4):191-195. 
22. Malhotra A, Chhaya N, Nsiah-Sarbeng P, et al. CT-guided deep inferior epigastric perforator (DIEP) flap localization -- better for the patient, the surgeon, and the hospital. Clin Radiol. 2013;68(2):131-138.
23. Rozen WM, Ashton MW, Whitaker IS, et al. The financial implications of computed tomographic angiography in DIEP flap surgery: a cost analysis. Microsurgery. 2009;29(2):168-199. 